\title{
Work extraction and fully entangled fraction
}

\author{
Chung-Yun Hsieh ${ }^{1, \text { * }}$ and Ray-Kuang Lee ${ }^{1,2}$ \\ ${ }^{1}$ Department of Physics, National Tsing Hua University, Hsinchu 300, Taiwan \\ ${ }^{2}$ Physics Division, National Center for Theoretical Sciences, Hsinchu 300, Taiwan
}

(Dated: August 27, 2018)

\begin{abstract}
For a bipartite state with equal local dimension $d$, we prove that one can obtain work gain under Landauer's erasure process on one party in identically and independently distributed (iid) limit when the corresponding fully entangled fraction is larger than $\frac{1}{d}$. By processing a given state to the maximally mixed state, we give an approximation for the largest extractable work with an error in the energy scale, which becomes negligible in the large system limit. As a step to link quantum thermodynamics and quantum nonlocality, we also provide a simple picture to approximate the optimal work extraction and suggest a potential thermodynamic interpretation of fully entangled fraction for isotropic states.
\end{abstract}

\section{INTRODUCTION}

Quantum thermodynamics and quantum nonlocality share similar spirit to tell quantum and classical regimes apart. For quantum nonlocality, we have the famous Einstein-Podolsky-Rosen (EPR) paradox [1] and Bell's inequality [2] to illustrate the bizarre nature of quantum theory. On the other hand, for quantum thermodynamics, a multitude of intriguing phenomena related to various definitions of work has been addressed [3]. Examples such as Landauer's principle [4, 5], different scenarios on work extraction [6 [8], the minimal work cost of running different processes such as a completely-positive tracepreserving map (CPTPM) 9, 10], or an erasure process [11], and other related results [12 18] simply certify the importance in this direction.

Even though the awareness of the relations between work and entanglement 19], coherence [20, 21], or other quantum correlations [14, 16, 22] have already been studied in the literature, a quantitative connection to nonlocal properties such as quantum nonlocality [23], quantum steerability 24, 25, and the usefulness of teleportation [26 29] still remains as an open question deserving further study. To this end, we adapt a well-known quantity called fully entangled fraction (FEF) [28, 29], which acts as a useful measurement in characterizing different nonlocal properties [23, 30 33]. In this work, we try to bridge quantum thermodynamics and quantum nonlocality together, by relating work gain under different processes to FEF.

With the help of FEF, for a given bipartite quantum state $\rho$ acting on $\mathbb{C}^{d} \otimes \mathbb{C}^{d}$, we derive an inequality to conclude that in identically and independently distributed (iid) limit, it is always possible to have work gain (i.e. negative work cost) of an erasure process on one party of $\rho$, provided that $\rho$ has FEF larger than $\frac{1}{d}$. This result gives an alternative interpretation for every state useful for teleportation [26, 28], which is equivalent to

*Electronic address: s103022502@m103.nthu.edu.tw enabling work gain under local erasure process. For a specified temperature $T$, we define work extraction to be a process mapping the initial state with fully degenerate system Hamiltonian to the final Gibbs state in $T$ with the Hamiltonian equal to the initial one. With some prerequisites, we obtain an approximation for the largest extractable work, up to an error in the energy scale which can be dropped when the system is large enough. This approximation not only provides a clear strategy for work extraction, which is consisting of two local operations and classical communications (LOCCs) plus one local erasure process, but also suggests us to interpret FEF for isotropic states [28] as a concept equivalent to the minimal work cost of erasure process on one party.

This paper is structured as follows. The notations used and the underline scenario to define erasure process and work extraction are described in Sec. [II. Then, in Sec. III, the first main result (Theorem III.2) is illustrated and proved, which gives an inequality linking FEF and work gain of local erasure process. The saturation for this inequality is discussed in Sec. IV] as a corollary of the approximation of the optimal extractable work (Theorem IV.1). Further more, we apply this result to isotropic states and arbitrary pure states in the remaining part of Sec. [V] where the former suggests us a thermodynamic interpretation of FEF, and the latter proposes a nearly-local protocol to extract the maximal work of an arbitrary pure state. Finally, Sec. $\nabla$ gives the conclusion.

\section{PRELIMINARY NOTIONS}

Through out this paper, we will only consider quantum states $\rho$ acting on a bipartite system, $\mathbb{C}^{d} \otimes \mathbb{C}^{d}$, with equal local dimension $d=2^{l}$ for some natural number $l \in \mathbb{N}$. For such a system, we call the first party "Alice" and the second party "Bob". The collection of all quantum states acting on this bipartite system is denoted as $\mathcal{L}\left(\mathbb{C}^{d} \otimes \mathbb{C}^{d}\right)$. The conditional von Neumann entropies (see, for exam- 
ple, Ref. [34] $)$ of a given state $\rho \in \mathcal{L}\left(\mathbb{C}^{d} \otimes \mathbb{C}^{d}\right)$ are:

$$
\begin{aligned}
& S(A \mid B)_{\rho}:=S(\rho)-S\left(\rho_{\mathrm{B}}\right) ; \\
& S(B \mid A)_{\rho}:=S(\rho)-S\left(\rho_{\mathrm{A}}\right) ;
\end{aligned}
$$

where $S(\rho):=-\operatorname{tr}\left(\rho \log _{2} \rho\right)$ is the von Neumann entropy of the quantum state $\rho$, and $\rho_{\mathrm{A}}:=\operatorname{tr}_{\mathrm{B}}(\rho)\left[\rho_{\mathrm{B}}:=\operatorname{tr}_{\mathrm{A}}(\rho)\right]$ is the local state on Alice's (Bob's) side.

The fully entangled fraction (FEF) [28, 29] of a given state $\rho \in \mathcal{L}\left(\mathbb{C}^{d} \otimes \mathbb{C}^{d}\right)$ is defined by

$$
\mathcal{F}(\rho):=\max _{|\Psi\rangle}\langle\Psi|\rho| \Psi\rangle
$$

where the maximization is taken over all maximally entangled states $|\Psi\rangle \in \mathbb{C}^{d} \otimes \mathbb{C}^{d}$. It is well known that FEF has been successfully applied to characterize different nonlocal properties. For instance, a quantum state $\rho \in \mathcal{L}\left(\mathbb{C}^{d} \otimes \mathbb{C}^{d}\right)$ is useful for the standard teleportation if and only if $\mathcal{F}(\rho)>\frac{1}{d}$ [28]; while $\rho$ is $k$-copy nonlocal (and hence $k$-copy steerable) if $\mathcal{F}(\rho)>\frac{1}{d}$ [31]. Sufficient conditions for quantum steerability can also be given by FEF [32, 33]. Therefore, if one is able to link FEF with quantum thermodynamics, it can be a good starting point for bridging quantum nonlocality and quantum thermodynamics.

For a given state $\rho \in \mathcal{L}\left(\mathbb{C}^{d} \otimes \mathbb{C}^{d}\right)$, we want to consider extractable work from the information content of $\rho$. More precisely, we tend to define work extraction by asking how much work can be extracted from $\rho$ by processing it to the maximally mixed state $\frac{\mathbb{I}}{d^{2}}$ ? The reason to adapt this definition is because we expect the maximally mixed state to contain the least useful information content. Therefore processing $\rho$ to it should be a natural way to obtain the optimal amount of work from the useful information possessed by $\rho$. To study work extraction and other processes, rigorously, we need to specify our framework, which is inspired by Ref. [11]. Let us consider the system $\mathbf{S}=\mathbb{C}^{d} \otimes \mathbb{C}^{d}$ couples to an environment $\mathbf{E}$, so that $\mathbf{S} \otimes \mathbf{E}$ is a closed system with only unitary time evolution. We require the initial system Hamiltonian to be fully degenerate. This means any computational basis will be energy eigenbasis; in particular, we are allowed to choose the computational basis in the form $\left\{\left|n_{1}\right\rangle \otimes\left|n_{2}\right\rangle \otimes \ldots \otimes\left|n_{l}\right\rangle \mid n_{1}, n_{2}, \ldots, n_{l} \in\{0,1\}\right\}$, where $\{|0\rangle,|1\rangle\}$ is a single qubit computational basis. The environment is consisting of two parts: a heat bath $\mathbf{B}_{T}$ in a finite and positive temperature $T$, and an observer $\mathbf{O}$. We further assume the observer possesses a subsystem, called a battery, which is assumed to be a perfect energy storage to store or withdraw energy without loss. The battery is also assumed to be able to generate energy in the form of work. For example, it can be a collection of work qubits [6] or a suspended weight that will be raised or lowered [7, 12]. In this sense, if energy is extracted from the system by the observer and stored in the battery, one can output the same amount of work from the battery. Theoretically and ideally, it is in this sense for us to talk about "work".
With the above setting, we can now consider the process that may help us to draw work from the system. For this purpose, we introduce the allowed actions for the observer $\mathbf{O}$ to apply, which amounts to observer's abilities to manipulate the system:

(1) Raising/Lowering energy level. This action allows the observer to raise or lower the energy level of the system (e.g. by adjusting the magnetic field), which is a crucial action for work extraction. If the system in an energy level $n$ and the observer changes the corresponding energy from $E_{n}$ to $E_{n}^{\prime}$, we define the work gain (for the observer) to be $E_{n}^{\prime}-E_{n}$ [11]. Note that we call it work gain based on the assumption of the existence of the ideal battery. This definition can be generalized to arbitrary states $\rho \in \mathcal{L}\left(\mathbb{C}^{d} \otimes \mathbb{C}^{d}\right)$ by $\operatorname{tr}\left[\rho\left(H^{\prime}-H\right)\right]$, where $H$ is the initial system Hamiltonian, and $H^{\prime}$ is the final Hamiltonian after this action, i.e., raising/lowering manipulation. We use $\mathcal{A}_{\mathrm{R} / \mathrm{L}}$ to denote the set of all actions of this type.

(2) Thermalization. Within this action, the observer will make (part of) the system couple to the heat bath $\mathbf{B}_{T}$ to achieve thermalization, and then the system will decouple from $\mathbf{B}_{T}$ in the end of this action. This amounts to some CPTPMs on the system, which is mainly used to make the system go to the Gibbs state. Although a CPTPM does need work cost [10], we assume that this cost is covered by the heat bath rather than the observer. Hence, we assume no energy change of the observer in this action. We use $\mathcal{A}_{T}$ to denote the set of all actions of this type with temperature $T$.

(3) Unitary operations. This action includes mappings in the form $\rho \mapsto \int_{U\left(d^{2}\right)} U \rho U^{\dagger} P_{U} d U$ for some probability density function $P_{U}$ over the unitary group $U\left(d^{2}\right)$. Also, we allow the observer to select specific results and abandon the rest, resulting in non-zero failure probability. Note that unlike the actions of thermalization, this action needs work cost from the observer [10]. In case of negative work cost, we define it to be work gain (upon success) given the existence of the ideal battery. We use $\mathcal{A}_{U}$ to denote the set of all actions of this type.

Apart from the actions of unitary operations, we also allow the following source of probabilistic nature, which is a special action for observer:

(4) $\delta$-approximation. Within this action, the observer is allowed to approximate the original input state by another state which is $\delta$-closed to it (a state $\rho$ is $\delta$-closed to another state $\sigma$ if their trace distance [9] is upper bounded by $\delta$, i.e., $\frac{1}{2}\|\rho-\sigma\|_{1} \leq \delta$ ). This conceptual action has no real effect on the state, and it is introduced so that in our framework one can define allowed processes rigorously and completely. Note that this step will result in nonzero failure probability. We use $\mathcal{A}_{\delta}$ to denote the set of all actions with $\delta$-precision of this type.

Now, we are in position to define process in our framework: for a given state $\rho \in \mathcal{L}\left(\mathbb{C}^{d} \otimes \mathbb{C}^{d}\right)$, we define an allowed process for $\rho$, denoted by $\mathcal{P}_{\rho}$, to be a finite sequence of actions $\mathcal{P}_{\rho}=\left\{A_{n}\right\}_{n=1}^{N} \subset \mathcal{A}_{\mathrm{R} / \mathrm{L}} \cup \mathcal{A}_{T} \cup \mathcal{A}_{U} \cup \mathcal{A}_{\delta}$ such that its behavior on the system can be mathemati- 
cally written as $A_{N} \circ A_{N-1} \circ \ldots \circ A_{1}$, where $A_{1}$ is acting on $\rho$ with the initial system Hamiltonian $H$ (fully degenerate), and $A_{N}$ will output a final state with final system Hamiltonian equal to the original one, i.e. $H$. Note that due to this definition, the observer can apply one and only one action at one time. To illustrate our idea, let us consider the proof of Theorem I.1 in Ref. [11] as an example. In their proof, in order to construct an erasure process on the given state $\rho$, they firstly choose a subsystem which is $\delta$-closed to a maximally entangled pure state $|\Psi\rangle$, and then they apply work extraction "for $|\Psi\rangle$ " on the given system. The work extraction they used for $|\Psi\rangle$ is consisting of actions in $\mathcal{A}_{R / L} \cup \mathcal{A}_{T}$ only (see Fig. 4 in Ref. [11]). In our framework, such process can therefore be written as $\mathcal{P}_{\rho}=\left\{A_{n}\right\}_{n=1}^{N}$, where $A_{1} \in \mathcal{A}_{\delta}$ and $\left\{A_{n}\right\}_{n=2}^{N} \subset \mathcal{A}_{R / L} \cup \mathcal{A}_{T}$. As mentioned previously, action of $\delta$-approximation results in probabilistic nature. This can be seen by the failure probability for the above process, which is less than $\delta$ as proved by Supplimentary Lemma IV.3 in Ref. [11].

Now, we define $W_{A}(\rho)$ to be the work gain for an action $A$ on $\rho$ upon success. Following this definition, we can define work gain of an allowed process $\mathcal{P}_{\rho}=\left\{A_{n}\right\}_{n=1}^{N}$, denoted by $W\left(\mathcal{P}_{\rho}\right)$, to be $W\left(\mathcal{P}_{\rho}\right):=\sum_{n=1}^{N} W_{A_{n}}(\rho)$, upon success of all the involved actions. This also explains why we define an allowed process to be a chain of actions rather than a single mapping: if one define a process to be a mapping, it may be ill-defined physically: there may be different chains of actions with different net work gains result in the same mapping, which is, however, not the physical property that we expect.

\section{LOCAL ERASURE PROCESS AND FULLY ENTANGLED FRACTION}

Consider a given quantum state $\rho \in \mathcal{L}\left(\mathbb{C}^{d} \otimes \mathbb{C}^{d}\right)$. Within our framework introduced in Sec. [II] we say $\mathcal{P}_{\rho}^{\mathrm{Er} \mid \mathrm{A}}$ is a local (Landauer's) erasure process for $\rho$ on $\mathrm{Al}-$ ice's side if (1) $\mathcal{P}_{\rho}^{\mathrm{Er} \mid \mathrm{A}}=\mathcal{P}_{|\phi\rangle\langle\phi|}$, where $|\phi\rangle\langle\phi|$ is a purification of $\rho$ (and the additional space needed for the purification is assumed to be included in the space of the observer), and (2) $\mathcal{P}_{|\phi\rangle\langle\phi|}$ maps $|\phi\rangle\langle\phi|\mapsto| \mathbf{0}\rangle\left\langle\mathbf{0}\left|\otimes \operatorname{tr}_{\mathrm{A}}\right| \phi\right\rangle\langle\phi|$, i.e., the state outside Alice's local system will be preserved. Here, we use the notation $|\mathbf{0}\rangle\langle\mathbf{0}|:=(|0\rangle\langle 0|)^{\otimes l}$. This shows why we call it a local erasure process. Also, we only consider minimal deterministic average work cost in iid limit of erasure process defined as follows [11] (note that we have a minus sign here because we always reserve positive value for work gain):

$$
\begin{aligned}
-W_{\operatorname{Er}}(\rho):=\inf \{ & w \mid \exists\left\{\mathcal{P}_{\rho^{\otimes k}}^{\operatorname{Er} \mid \mathrm{A}}\right\}_{k=1}^{\infty} \text { s.t. } \\
& \left.\lim _{k \rightarrow \infty} P\left[-W\left(\mathcal{P}_{\rho^{\otimes k}}^{\mathrm{Er} \mid \mathrm{A}}\right) \leq k w\right]=1\right\},
\end{aligned}
$$

where $P[-W(\mathcal{P}) \leq x]$ is the probability for the process $\mathcal{P}$ to achieve work cost $-W(\mathcal{P})$ no greater than $x$ (recall the probabilistic nature of allowed actions). To understand the meaning of this definition, note that only the regime $k \gg 1$ will be important. Hence, $-W_{\operatorname{Er}}(\rho) \times k$ is the minimal work cost that can be achieved almost with certainty for any large enough $k$, and the probability of failure will go to zero when $k \rightarrow \infty$. This justifies the interpretation of minimal deterministic average work cost in iid limit. From Ref. [11], we have the following upper bounds:

$$
-W_{\mathrm{Er}}(\rho) \leq S(A \mid B)_{\rho} k_{B} T \ln 2 .
$$

The remarkable consequence of the above inequality is the existence of negative work cost of local erasure process for some entangled states [11], which also implies that $W_{\operatorname{Er}}(\rho)>0$ serves as an entanglement witness. However, how strong the entanglement needs to be in order to have work gain under local erasure process is still unknown. This is one example of questions that can be answered by our first main result, which is a direct consequence of the following lemma, whose proof can be found in Appendix A

Lemma III.1. If $\mathcal{F}(\rho)>\frac{1}{d}$, then

$$
S(A \mid B)_{\rho} \leq-\log _{2} \mathcal{F}(\rho) d .
$$

Combining Eq. (4) and Lemma III.1 we obtain our first main result:

Theorem III.2. If $\mathcal{F}(\rho)>\frac{1}{d}$, then

$$
W_{\mathrm{Er}}(\rho) \geq k_{B} T \ln \mathcal{F}(\rho) d .
$$

In other words, states with FEF larger than $\frac{1}{d}$ enable work gain under local erasure process.

\section{A. Theorem III.2 and Teleportation}

Several remarks can be made accordingly. As the first one, we recall the famous result developed by Holodecki et al. [28] that a given state $\rho \in \mathcal{L}\left(\mathbb{C}^{d} \otimes \mathbb{C}^{d}\right)$ is useful for teleportation if and only if $\mathcal{F}(\rho)>\frac{1}{d}$. Together with Theorem [II.2, one learn that a state $\rho \in \mathcal{L}\left(\mathbb{C}^{d} \otimes \mathbb{C}^{d}\right)$ admits deterministic work gain in iid limit under local erasure process if it is useful for teleportation. This build a simple while quantitative link between erasure process and the teleportation power. Also, Ref. 11] shows that $W_{\mathrm{Er}}(\rho)>0$ is a sufficient condition of entanglement. Via Theorem III.2, one is able to partially address the converse - we learn that $\mathcal{F}(\rho)>\frac{1}{d}$ is a sufficient condition of $W_{\operatorname{Er}}(\rho)>0$. This means when the entanglement is strong enough (in the sense that FEF $>\frac{1}{d}$ ), there exists a local erasure process inducing work gain.

\section{B. Theorem III.2 and Nonlocal Properties}

As another remark, Theorem III.2 can be used to establish sufficient condition of locality and nonsteerability for certain class of quantum states with FEF 
$>\frac{1}{d}$. To see this, we note that Theorem III.2 implies the following upper bound on the FEF of the given state $\rho$ with $\mathcal{F}(\rho)>\frac{1}{d}$ :

$$
\mathcal{F}(\rho) \leq \frac{1}{d} e^{\frac{W_{\mathrm{Er}}(\rho)}{k_{B} T}}
$$

As an example, consider isotropic state [28] defined by

$$
\rho_{\text {iso }}(p):=p\left|\Psi_{d}^{+}\right\rangle\left\langle\Psi_{d}^{+}\right|+(1-p) \frac{\mathbb{I}}{d^{2}},
$$

where $\left|\Psi_{d}^{+}\right\rangle:=\frac{1}{\sqrt{d}} \sum_{i=0}^{d-1}|i i\rangle$ is the generalized singlet and $p \in\left[-\frac{1}{d^{2}-1}, 1\right]$ due to the positivity of a quantum state. As a direct corollary, since $\rho_{\text {iso }}$ is entangled if and only if $\mathcal{F}\left(\rho_{\text {iso }}\right)>\frac{1}{d}$ [23], Theorem III.2 implies $\rho_{\text {iso }}$ admits work gain under local erasure process if and only if $\rho_{\mathrm{iso}}$ is entangled. For other nonlocal correlations, we make use the well-known property of isotropic states: there exist many thresholds for different nonlocal properties [23]. For instance, there exist $\mathcal{F}_{\text {LHV }}, \mathcal{F}_{\text {LHS }}^{\pi}$, and $\mathcal{F}_{\text {LHS }}$ such that $\rho_{\text {iso }}$ is local under general positive operator-value measures (POVMs) if $\mathcal{F}\left(\rho_{\text {iso }}\right) \leq \mathcal{F}_{\text {LHV }}[23,36] ; \rho_{\text {iso }}$ is unsteerable under projective POVMs if and only if $\mathcal{F}\left(\rho_{\text {iso }}\right) \leq \mathcal{F}_{\text {LHS }}^{\pi}=\frac{H_{d}+H_{d} d-d}{d^{2}}$, where $H_{d}:=\sum_{n=1}^{d} \frac{1}{n}$ [25], and $\rho_{\text {iso }}$ is unsteerable under general POVMs if $\mathcal{F}\left(\rho_{\text {iso }}\right) \leq \mathcal{F}_{\text {LHS }}=\tilde{p}_{\phi}\left(1-\frac{1}{d^{2}}\right)+\frac{1}{d^{2}}$, where $\tilde{p}_{\phi}:=\frac{3 d-1}{d^{2}-1}\left(1-\frac{1}{d}\right)^{d}$ [36]. Applying Eq. (17) on $\rho_{\text {iso }}$ with $\mathrm{FEF}>\frac{1}{d}$, we learn that it is local if

$$
W_{\mathrm{Er}}\left(\rho_{\text {iso }}\right) \leq k_{B} T \ln \mathcal{F}_{\mathrm{LHV}} d
$$

it is unsteerable under general POVMs if

$$
W_{\mathrm{Er}}\left(\rho_{\text {iso }}\right) \leq k_{B} T \ln \left[\tilde{p}_{\phi}\left(d-\frac{1}{d}\right)+\frac{1}{d}\right]
$$

it is unsteerable under projective POVMs if

$$
W_{\mathrm{Er}}\left(\rho_{\text {iso }}\right) \leq k_{B} T \ln \frac{H_{d}+H_{d} d-d}{d} .
$$

Because there is a hierarchy consisting of different thresholds for FEF of isotropic states, one may wonder whether Eq. (7) can map this hierarchy onto the one consisting of different erasure work costs. This can be achieved if the upper bound in Eq. (7) is saturated by isotropic states. We will back to this issue in Sec. IVA.

\section{WORK EXTRACTION PROCESS AND FULLY ENTANGLED FRACTION}

In this section, we want to study relation between work extraction and fully entangled fraction. Before proceeding, let us firstly define the former. Consider a given state $\rho \in \mathcal{L}\left(\mathbb{C}^{d} \otimes \mathbb{C}^{d}\right)$. We say $\mathcal{P}_{\rho}^{\mathrm{W}}$ is a work extraction process for $\rho$ if it is an allowed process having $\frac{\mathbb{I}}{d^{2}}$ as the final state. Note that the final Hamiltonian is also fully degenerate in our formalism, which means that the maximally mixed state equals the Gibbs state in the given temperature. Due to this definition, we define the largest deterministic extractable work in iid limit for $\rho$ as:

$$
\begin{aligned}
W_{\text {Total }}(\rho):=\sup \{ & w \mid \exists\left\{\mathcal{P}_{\rho}^{\mathrm{W}}\right\}_{k=1}^{\infty} \text { s.t. } \\
& \left.\lim _{k \rightarrow 1} P\left[W\left(\mathcal{P}_{\rho \otimes k}^{\mathrm{W}}\right) \geq k w\right]=1\right\} .
\end{aligned}
$$

Just like the definition of erasure work cost, $W_{\text {Total }}(\rho)$ serves as the maximal work gain from $\rho$ with certainty in iid limit. This definition should be an answer of our initial question in iid limit, i.e., how much work can be extracted by processing $\rho$ to $\frac{\mathbb{I}}{d^{2}}$.

As a direct observation from Eq. (4), since the composition of processes $\mathcal{P}_{(|\mathbf{0}\rangle\langle\mathbf{0}|) \otimes k}^{\mathrm{W}} \circ \mathcal{P}_{\rho^{\otimes k}}^{\mathrm{Er}}$ is a work extraction process, we conclude that (see Appendix Cfor the proof)

$$
W_{\text {Total }}(\rho) \geq k_{B} T \ln d^{2}-S(\rho) k_{B} T \ln 2 .
$$

Before stating the main result, we still need to introduce an estimate done by Dahlsten et al. [12]. Define an $\epsilon$-compression action for $\rho$ to be an action of unitary operations mapping as $\rho \mapsto U \rho U^{\dagger}$ such that $U \rho U^{\dagger}$ is $2 \epsilon$-close to a state of the form $\rho^{\prime} \otimes|\psi\rangle\langle\psi|$, with success probability $P_{\text {success }} \geq 1-2 \epsilon[35]$. Then the process adapted by Ref. [12] is given by $\left\{A_{n}\right\}_{n=1}^{N}$, where $A_{1}$ is an $\epsilon$-compression action, and $\left\{A_{n}\right\}_{n=2}^{N}$ amounts to a work extraction process on $|\psi\rangle\langle\psi|$ with work gain $k_{B} T \ln d_{\psi}$, where $d_{\psi}$ is the dimension of the local system $|\psi\rangle\langle\psi|$. We call them compression-extraction processes, and use the notation $\mathcal{P}_{\rho}^{\epsilon}$ to denote such processes with $P_{\text {success }} \geq$ $1-2 \epsilon$. Then Theorem 2 in Ref. [12] states that if the extractable work by a compression-extraction process of a given bipartite state $\rho \in \mathcal{L}\left(\mathbb{C}^{d} \otimes \mathbb{C}^{d}\right)$ is lower bounded by $k_{B} T \ln d^{2}-\left[H_{\min }^{\epsilon}(\rho)+3 \ln \epsilon\right] k_{B} T \ln 2$, then we have $P_{\text {success }}<2 \epsilon$. Here $H_{\min }^{\epsilon}(\rho)$ is the smooth min-entropy of $\rho$, whose definition can be found in Eq. (D3) (we refer the readers to Refs. [12, 34] and references therein for the detail). This means whenever one is able to choose some $\mathcal{P}_{\rho}^{\frac{1}{2}-\epsilon}$ to extract $W_{\text {Total }}(\rho)$ (therefore with $P_{\text {success }} \geq 2 \epsilon$ ), Theorem 2 in Ref. 12] becomes an upper bound on it: $W_{\text {Total }}(\rho)<k_{B} T \ln d^{2}-\left[H_{\min }^{\epsilon}(\rho)+3 \ln \epsilon\right] k_{B} T \ln 2$. With Lemma III.1 in hand, we are now able to estimate $W_{\text {Total }}(\rho)$ in terms of FEF and conditional von Neumann entropy under the above conditions: [We refer the reader to Appendix $\mathrm{D}$ for the proof; also, we adapt the notation $\left.S_{\min }(\rho):=\min \left\{S\left(\rho_{\mathrm{A}}\right) ; S\left(\rho_{\mathrm{B}}\right)\right\}\right]$

Theorem IV.1. Given $0<\epsilon \leq \frac{1}{2}$ and $\delta_{\epsilon}:=-3 \ln \epsilon$. If (1) $\mathcal{F}(\rho)>\frac{1}{d}$,

(2) there exits $\mathcal{P}_{\rho}^{\frac{1}{2}-\epsilon}$ which can extract $W_{\text {Total }}(\rho)$, and

(3) $\log _{2}\|\rho\|_{\infty}=\log _{2} \mathcal{F}(\rho) d-S_{\min }(\rho)$, then

$$
W_{\text {Total }}(\rho) \approx k_{B} T \ln d^{2}-S_{\min }(\rho) k_{B} T \ln 2+W_{\operatorname{Er}}(\rho),(
$$

and

$$
W_{\mathrm{Er}}(\rho) \approx k_{B} T \ln \mathcal{F}(\rho) d,
$$

up to an error $\delta_{\epsilon} k_{B} T \ln 2$. 


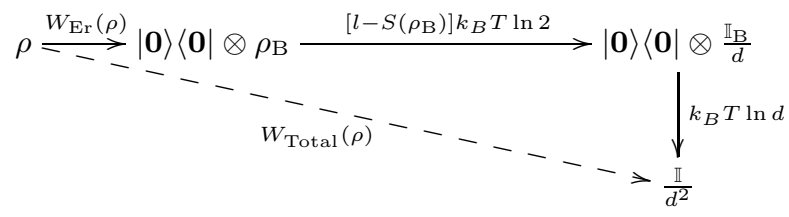

FIG. 1: Schematic interpretation of Eq. (14). Assume $S_{\min }(\rho)=S\left(\rho_{\mathrm{B}}\right)$ without loss of generality. In this diagram, via an optimal local erasure process in iid limit on Alice's side (the upper-left arrow), a work extraction on Bob's side (the upper-right arrow), and a work extraction on Alice's side (the downward arrow), $W_{\text {Total }}(\rho)$ is extracted (the dashed arrow), up to $\delta_{\epsilon} k_{B} T \ln 2$.

As a remark, Eq. (14) provides a picture (see Fig. 1) of an approximately optimal global deterministic work extraction in iid limit. Note that the above approximation will be faithful if $\delta_{\epsilon} k_{B} T \ln 2 \ll k_{B} T \ln d$, i.e., if $\delta_{\epsilon} \ll l$, where $l$ is the number of qubits in a single party $\left(d=2^{l}\right)$. In other words, $\delta_{\epsilon} k_{B} T \ln 2$, which is the best resolution for energy in this case, will be an irrelevant scale. Theorem IV.1 can be regarded as an approximately sufficient condition of the tightness of Theorem III.2, and thus Eq. (4) and Lemma III.1. In particular, approximate saturation for Lemma III.1 together with condition (3) in the above theorem implies the following corollary:

$$
S(\rho) \approx-\log _{2}\|\rho\|_{\infty},
$$

up to an error $\delta_{\epsilon}$.

To illustrate the applications of Theorem IV.1, let us compute some examples. In particular, what we want is a thermodynamic interpretation of FEF for certain quantm states. Due to later consideration, let us define $\Lambda(\rho):=\log _{2}\|\rho\|_{\infty}-\left[\log _{2} \mathcal{F}(\rho) d-S_{\min }(\rho)\right]$. The following subsections hold for $\epsilon$-values satisfying TheoremIV.1.

\section{A. Theorem IV.1 and Isotropic States}

From Eq. (8), one can see that $\Lambda\left[\rho_{\text {iso }}(p)\right]=0$ for all $p$ [to prove this, it suffices to note that $\mathcal{F}\left(\rho_{\text {iso }}\right)=\left\|\rho_{\text {iso }}\right\|_{\infty}$ and $\left.S_{\min }\left(\rho_{\text {iso }}\right)=\log _{2} d\right]$. This means for isotropic states with FEF $>\frac{1}{d}$, if there exists $\mathcal{P}_{\rho_{\text {iso }}}^{\frac{1}{2}-\epsilon}$ which can extract $W_{\text {Total }}\left(\rho_{\text {iso }}\right)$, then $W_{\text {Total }}\left(\rho_{\text {iso }}\right) \approx k_{B} T \ln d+W_{\text {Er }}\left(\rho_{\text {iso }}\right)$ and $W_{\text {Er }}\left(\rho_{\text {iso }}\right) \approx k_{B} T \ln \mathcal{F}\left(\rho_{\text {iso }}\right) d$ up to an energy scale $\delta_{\epsilon} k_{B} T \ln 2$. In particular, the latter suggests a possible thermodynamic interpretation of FEF for isotropic states-up to $\delta_{\epsilon} k_{B} T \ln 2$, FEF of isotropic states is a concept equivalent to the minimal deterministic work cost in iid limit of local erasure process.

Also note that $W_{\mathrm{Er}}\left(\rho_{\text {iso }}\right) \approx k_{B} T \ln \mathcal{F}\left(\rho_{\text {iso }}\right) d$ builds an approximate hierarchy according to the result discussed in Sec. IIIB from the approximate saturation of Eq. (7); namely, by substituting different FEF thresholds of nonlocal properties for isotropic states into the saturation

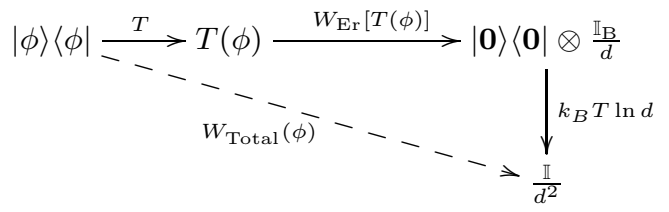

FIG. 2: Schematic interpretation for the optimal deterministic work extraction in iid limit up to $\delta_{\epsilon} k_{B} T \ln 2$. The upperleft arrow is the quantum twirling bringing the input pure state to an isotropic state, and the remaining processes, i.e. the upper-right and downward arrows, are just the consequence of Eq. (14) on isotropic states.

bound, one obtain the corresponding erasure work cost threshold for isotropic states, up to the energy scale $\delta_{\epsilon} k_{B} T \ln 2$. For example, if $\rho_{\text {iso }}$ (with FEF $>\frac{1}{d}$ ) can achieve the equality in Eq. (7), then Eq. (11) implies $\rho_{\text {iso }}$ is steerable under projective measurement if and only if $W_{\mathrm{Er}}\left(\rho_{\text {iso }}\right) \leq k_{B} T \ln \frac{H_{d}+H_{d} d-d}{d}$. Being saturated with a resolution $\delta_{\epsilon} k_{B} T \ln 2$, we may interpret $k_{B} T \ln \frac{H_{d}+H_{d} d-d}{d}$ as an approximate energy threshold for isotropic states with possible error $\delta_{\epsilon} k_{B} T \ln 2$, which will be small when the system is large enough. Similar argument applies to other thresholds found in Sec. IIIB.

\section{B. Theorem IV.1 and Arbitrary Pure States}

Now let us consider arbitrary pure states $|\phi\rangle \in \mathbb{C}^{d} \otimes \mathbb{C}^{d}$ (we adapt the notation $\phi:=|\phi\rangle\langle\phi|$ ). From Ref. [28], we learn that every state $\rho \in \mathcal{L}\left(\mathbb{C}^{d} \otimes \mathbb{C}^{d}\right)$ can be turned into an isotropic state via quantum twirling

$$
T(\rho):=\int_{U(d)}\left(U \otimes U^{*}\right) \rho\left(U \otimes U^{*}\right)^{\dagger} d U
$$

where $d U$ is the Haar measure representing uniform distribution over the unitary group $U(d)$. Now, the minimal work cost of the quantum twirling on $\phi$ is given by [10] $k_{B} T \ln \left\|T\left(\Pi_{\phi}\right)\right\|_{\infty}$, where $\Pi_{\phi}$ is the projector onto the support of the state $\phi$. Because $\phi$ is pure, we have $\Pi_{\phi}=\phi$. Since $\left\|\rho_{\text {iso }}\right\|_{\infty}=\mathcal{F}\left(\rho_{\text {iso }}\right)$ and $T(\phi)$ is an isotropic state, the minimal work cost of quantum twirling is $k_{B} T \ln \mathcal{F}[T(\phi)]$. By assuming the existence of $\mathcal{P}_{T(|\phi\rangle\langle\phi|)}^{\frac{1}{2}-\epsilon}$ which can extract $W_{\text {Total }}[T(|\phi\rangle\langle\phi|)]$, we use the result for isotropic state to conclude the following approximation for pure states achieving $\mathcal{F}[T(\phi)]>\frac{1}{d}$ :

$$
\begin{aligned}
W_{\text {Total }}(\phi) & \approx-k_{B} T \ln \mathcal{F}[T(\phi)]+k_{B} T \ln \mathcal{F}[T(\phi)] d^{2} \\
& =k_{B} T \ln d^{2},
\end{aligned}
$$

up to the precision $\delta_{\epsilon} k_{B} T \ln 2$. This is the value predicted by Landauer's principle [5, 11, 16] (note that, however, this is still an approximation because the result for isotropic states is not exact). See Fig. 2 for the schematic interpretation. 


\section{CONCLUSION}

In this work, we try to connect quantum thermodynamics and quantum nonlocality. Consider a given state $\rho \in \mathcal{L}\left(\mathbb{C}^{d} \otimes \mathbb{C}^{d}\right)$ with dimension $d=2^{l}$ with $l \in \mathbb{N}$, we prove an inequality which shows that $\rho$ can induce work gain under local erasure process deterministic in iid limit if $\mathcal{F}(\rho)$, its fully entangled fraction (FEF), is larger than $\frac{1}{d}$, thereby connecting work gain under erasure process to the usefulness of quantum teleportation [28].

By considering work extraction under temperature $T$ as a process mapping the initial state $\rho$ with a fully degenerate Hamiltonian to Gibbs state in $T$ with the same Hamiltonian (therefore $\frac{\mathbb{I}}{d^{2}}$ ), we derive an approximation for the optimal deterministic extractable work in iid limit, with three prerequisites and an error in the energy scale, which is small in the large system limit. The prerequisites of this approximation also serve as a sufficient condition of approximate saturation of our first main result. Moreover, a simple picture of optimal work extraction process deterministic in iid limit is proposed by this approximation. When it is applicable to isotropic state, we further obtain a possible thermodynamic interpretation of FEF: up to an error in the energy scale, FEF of isotropic states is conceptually equivalent to the minimal work cost (deterministic in iid limit) of local erasure process. When we focus on pure states, an improved version of the approximation can be derived. The results we obtained can be a starting point of future research on the interface of quantum thermodynamics and quantum nonlocality.

\section{ACKNOWLEDGEMENTS}

This work was supported by the Ministry of Science and Technology of Taiwan under Grant No. 105-2119-M007-004. Authors acknowledge many fruitful discussions with Prof. Yeong-Cherng Liang and Prof. Hsiu-Hau Lin.

\section{Appendix A: Proof of Lemma III.1}

Proof. From Ref. [37], we learn that:

$$
\begin{aligned}
& S(A \mid B)_{\rho}=\lim _{\epsilon \rightarrow 0} \lim _{k \rightarrow \infty} \frac{1}{k} H_{\min }^{\epsilon}\left(A^{\otimes k} \mid B^{\otimes k}\right)_{\rho} \otimes k \\
& :=\lim _{\epsilon \rightarrow 0} \lim _{k \rightarrow \infty} \frac{1}{k} \sup _{\left\|\eta-\rho^{\otimes k}\right\|_{B}<\epsilon} H_{\min }\left(A^{\otimes k} \mid B^{\otimes k}\right)_{\eta},
\end{aligned}
$$

where $\|\rho-\sigma\|_{B}:=\sqrt{2-2 F(\rho, \sigma)}$ is the Bures distance [37] ( $F$ is the fidelity defined in Ref. [9] ). According to Theorem 2 in Ref. [37], we have

$$
H_{\min }(A \mid B)_{\rho}=-\log _{2}\left[Q(A \mid B)_{\rho} d\right]
$$

with

$$
Q(A \mid B)_{\rho}:=\max _{\mathcal{E}}\left\langle\Psi_{d}^{+}\left|\left(\mathbb{I}_{A} \otimes \mathcal{E}\right)(\rho)\right| \Psi_{d}^{+}\right\rangle
$$

where $\mathcal{E}: \mathcal{L}\left(\mathbb{C}^{d}\right) \rightarrow \mathcal{L}\left(\mathbb{C}^{d}\right)$ is a CPTPM. Substituting the above form, using Lemma B.2, and note the fact $Q\left(A^{\otimes k} \mid B^{\otimes k}\right)_{\rho \otimes k} \geq \mathcal{F}\left(\rho^{\otimes k}\right) \geq \mathcal{F}(\rho)^{k}$, we conclude

$$
\begin{aligned}
& S(A \mid B)_{\rho}=-\lim _{\epsilon \rightarrow 0} \lim _{k \rightarrow \infty} \frac{1}{k} \log _{2}\left[Q\left(A^{\otimes k} \mid B^{\otimes k}\right)_{\rho^{\otimes k}} d^{k}\right] . \\
& \leq-\lim _{\epsilon \rightarrow 0} \lim _{k \rightarrow \infty} \frac{1}{k} \log _{2}[\mathcal{F}(\rho) d]^{k}=-\log _{2} \mathcal{F}(\rho) d .
\end{aligned}
$$

\section{Appendix B: Notes on the $Q$ function}

To start with, let us define the Hilbert-Schmidt norm on an operator $X$ as the norm induced by the HilbertSchmidt inner product [9] :

$$
\|X\|_{2}:=\sqrt{\operatorname{tr}\left(X^{\dagger} X\right)}
$$

First, we prove the following lemma:

Lemma B.1. $Q(A \mid B)_{\rho}$ is continuous on $\rho \in \mathcal{L}\left(\mathbb{C}^{d} \otimes \mathbb{C}^{d}\right)$ with Hilbert-Schmidt norm.

Proof. Given $\|\rho-\sigma\|_{2}<\epsilon$, there exists a CPTPM $\mathcal{E}_{\epsilon}$ achieving [We choose $Q(A \mid B)_{\rho}=\left\langle\Psi_{d}^{+}\left|\left(\mathbb{I}_{\mathrm{A}} \otimes \mathcal{E}_{\epsilon}\right)(\rho)\right| \Psi_{d}^{+}\right\rangle$ when $Q(A \mid B)_{\rho} \geq Q(A \mid B)_{\sigma}$ and $Q(A \mid B)_{\sigma}=\left\langle\Psi_{d}^{+}\right|\left(\mathbb{I}_{\mathrm{A}} \otimes\right.$ $\left.\mathcal{E}_{\epsilon}\right)(\sigma)\left|\Psi_{d}^{+}\right\rangle$when $\left.Q(A \mid B)_{\rho} \leq Q(A \mid B)_{\sigma}\right]$

$$
\begin{aligned}
& \left|Q(A \mid B)_{\rho}-Q(A \mid B)_{\sigma}\right| \\
& \leq\left|\left\langle\Psi_{d}^{+}\left|\left(\mathbb{I}_{\mathrm{A}} \otimes \mathcal{E}_{\epsilon}\right)(\rho)\right| \Psi_{d}^{+}\right\rangle-\left\langle\Psi_{d}^{+}\left|\left(\mathbb{I}_{\mathrm{A}} \otimes \mathcal{E}_{\epsilon}\right)(\sigma)\right| \Psi_{d}^{+}\right\rangle\right| \\
& =\left|\left\langle\Psi_{d}^{+}\left|\left(\mathbb{I}_{\mathrm{A}} \otimes \mathcal{E}_{\epsilon}\right)(\rho-\sigma)\right| \Psi_{d}^{+}\right\rangle\right| \\
& =\left|\operatorname{tr}\left[\left(\mathbb{I}_{\mathrm{A}} \otimes \mathcal{E}_{\epsilon}^{\dagger}\right)\left(\left|\Psi_{d}^{+}\right\rangle\left\langle\Psi_{d}^{+}\right|\right)(\rho-\sigma)\right]\right| \\
& =\frac{1}{d}\left|\operatorname{tr}\left[\mathcal{J}\left(\mathcal{E}_{\epsilon}^{\dagger}\right)(\rho-\sigma)\right]\right|
\end{aligned}
$$

where $\mathcal{J}$ is the Choi-Jamiołkowski isomorphism 38]. Now, we use the following generalized Cauchy-Schwarz inequality of operators proved by Bhatia, which holds for arbitrary operators $X, Y$, and arbitrary unitarily invariant norms $\|\cdot\|[39,40]$ :

$$
\left\|\left|X^{\dagger} Y\right|^{\frac{1}{2}}\right\|^{2} \leq\|X\|\|Y\|
$$

where a norm $\|\cdot\|$ is unitarily invariant if $\|U X V\|=$ $\|X\|$ holds for all operator $\mathrm{X}$ and unitary operators $U$, $V$. Since $\|\cdot\|_{2}$ is unitarily invariant, direct computation shows that

$$
\begin{aligned}
\|X\|_{2}\|Y\|_{2} & \geq\left\|\left|X^{\dagger} Y\right|^{\frac{1}{2}}\right\|_{2}^{2} \\
& =\operatorname{tr}\left[\left|X^{\dagger} Y\right|^{\frac{1}{2}, \dagger}\left|X^{\dagger} Y\right|^{\frac{1}{2}}\right]=\operatorname{tr}\left|X^{\dagger} Y\right|
\end{aligned}
$$

This implies (note that the inequality $|\operatorname{tr}(A)| \leq \operatorname{tr}|A|$ 
holds for any operator $A^{1}$ )

$$
\begin{aligned}
& \frac{1}{d}\left|\operatorname{tr}\left[\mathcal{J}\left(\mathcal{E}_{\epsilon}^{\dagger}\right)(\rho-\sigma)\right]\right| \leq \frac{1}{d} \operatorname{tr}\left|\mathcal{J}\left(\mathcal{E}_{\epsilon}^{\dagger}\right)(\rho-\sigma)\right| \\
& \leq \frac{1}{d}\left\|\mathcal{J}\left(\mathcal{E}_{\epsilon}^{\dagger}\right)\right\|_{2}\|\rho-\sigma\|_{2} \leq \frac{1}{d}\|\rho-\sigma\|_{2}<\frac{\epsilon}{d} .
\end{aligned}
$$

Note that $\left\|\mathcal{J}\left(\mathcal{E}_{\epsilon}^{\dagger}\right)\right\|_{2} \leq 1$ since $\mathcal{J}\left(\mathcal{E}_{\epsilon}^{\dagger}\right)$ is a normalized state due to Choi-Jamiołkowski isomorphism theorem [38]. This completes the proof.

Now, we are in position to prove the following result:

Lemma B.2. If $\mathcal{F}(\rho)>\frac{1}{d}$, then for all $k \in \mathbb{N}$ and $\epsilon \in$ $(0,1)$, there exists $0 \leq o(\epsilon) \leq \log _{2}\left(\frac{2 \epsilon}{1-2 \epsilon}+1\right)$ such that

$$
\begin{aligned}
& \inf _{|| \eta-\rho^{\otimes k}||_{B}<\epsilon} \log _{2} Q\left(A^{\otimes k} \mid B^{\otimes k}\right)_{\eta} \\
& =\log _{2} Q\left(A^{\otimes k} \mid B^{\otimes k}\right)_{\rho^{\otimes k}}+o(\epsilon) .
\end{aligned}
$$

Proof. Consider a fixed $k$ value and a fixed $\epsilon \in(0,1)$. We first note the relation $\|\rho-\sigma\|_{2} \leq 2\|\rho-\sigma\|_{B}{ }^{2}$. This means $\left\|\eta-\rho^{\otimes k}\right\|_{2}<2 \epsilon$ if $\left\|\eta-\rho^{\otimes k}\right\|_{B}<\epsilon$. When $\left\|\eta-\rho^{\otimes k}\right\|_{B}<\epsilon$, Lemma B.1 implies

$$
\begin{aligned}
& \left|Q\left(A^{\otimes k} \mid B^{\otimes k}\right)_{\rho^{\otimes k}}-Q\left(A^{\otimes k} \mid B^{\otimes k}\right)_{\eta}\right| \\
& \leq \frac{1}{d^{k}}\left\|\rho^{\otimes k}-\eta\right\|_{2}<\frac{2 \epsilon}{d^{k}} .
\end{aligned}
$$

For $\rho$ with $\mathcal{F}(\rho)>\frac{1}{d}$, the function $Q\left(A^{\otimes k} \mid B^{\otimes k}\right)_{\rho^{\otimes k}}$ only takes values from the interval $\left(\frac{1}{d^{k}}, 1\right]$. Hence, if $|x-y|<\frac{2 \epsilon}{d^{k}}$ for some $x \in\left(\frac{1}{d^{k}}, 1\right]$ and $y \in\left(\frac{1-2 \epsilon}{d^{k}}, 1\right]$ (assume $x \geq y$ without loss of generality), we have $\left|\log _{2} x-\log _{2} y\right|<\log _{2}\left(\frac{2 \epsilon}{d^{k} y}+1\right)<\log _{2}\left(\frac{2 \epsilon}{1-2 \epsilon}+1\right)$. Substituting $x=Q\left(A^{\otimes k} \mid B^{\otimes k}\right)_{\rho \otimes k}$ and $y=Q\left(A^{\otimes k} \mid B^{\otimes k}\right)_{\eta}$ and consider $\left\|\eta-\rho^{\otimes k}\right\|_{B}<\epsilon$, we conclude

$$
\begin{aligned}
& \inf _{|| \eta-\left.\rho^{\otimes k}\right|_{B}<\epsilon} \log _{2} Q\left(A^{\otimes k} \mid B^{\otimes k}\right)_{\eta} \\
& =\log _{2} Q\left(A^{\otimes k} \mid B^{\otimes k}\right)_{\rho^{\otimes k}}-o(\epsilon)
\end{aligned}
$$

\footnotetext{
1 To prove $|\operatorname{tr}(A)| \leq \operatorname{tr}|A|$ for an arbitrary operator $A$, we firstly choose the polar decomposition [9] as $A=U J$, where $U$ is unitary and $J$ is positive. Then one can see it remains to prove $|\operatorname{tr}(U J)| \leq \operatorname{tr}(J)$, where $J$ is nothing but $|A|$. To show this, let us write the spectrum decomposition 9 as $J=\sum_{n} a_{n}\left|\phi_{n}\right\rangle\left\langle\phi_{n}\right|$, where $\left\{\left|\phi_{n}\right\rangle\right\}$ is an orthonormal basis of the given state space and $a_{n}>0 \forall n$. Then direct computation shows $|\operatorname{tr}(U J)|=\left|\sum_{n, m}\left\langle\phi_{m}\left|U b_{n}\right| \phi_{n}\right\rangle\left\langle\phi_{n} \mid \phi_{m}\right\rangle\right|=$ $\left|\sum_{n}\left\langle\phi_{n}|U| \phi_{n}\right\rangle b_{n}\right| \leq \sum_{n}\left|\left\langle\phi_{n}|U| \phi_{n}\right\rangle\right| b_{n} \leq \sum_{n} b_{n}=\operatorname{tr}(J)$.

2 Note that $\|\rho-\sigma\|_{2} \leq\|\rho-\sigma\|_{1} \leq 2 \sqrt{1-F(\rho, \sigma)^{2}} \leq$ $2 \sqrt{2[1-F(\rho, \sigma)]}=2|| \rho-\sigma \|_{B}[9]$, where $\|A\|_{1}:=\operatorname{tr}|A|$ is the trace norm. To see the fact $\|\rho-\sigma\|_{2} \leq\|\rho-\sigma\|_{1}$, we first apply polar decomposition [9] to write $\rho-\sigma=U J$, where $U$ is a unitary operator and $J$ is a positive operator. Then we have $\|\rho-\sigma\|_{2}=\|J\|_{2}$ and $\|\rho-\sigma\|_{1}=\|J\|_{1}$. Choose the spectrum decomposition [9] as $J=\sum_{n} a_{n}\left|\phi_{n}\right\rangle\left\langle\phi_{n}\right|$, where $\left\{\left|\phi_{n}\right\rangle\right\}$ is an orthonormal basis and $a_{n} \geq 0 \forall n$. One can verify that $\|J\|_{2}^{2}=\sum_{n} a_{n}^{2} \leq\left(\sum_{n} a_{n}\right)^{2}=\|J\|_{1}^{2}$, which proves the desired result.
}

with a function $o(\epsilon)$ satisfying $0 \leq o(\epsilon) \leq \log _{2}\left(\frac{2 \epsilon}{1-2 \epsilon}+1\right)$.

\section{Appendix C: Proof of Equation (13)}

Proof. First, we consider a fixed $\epsilon \in(0,1)$. For a given $k$, we choose $\Delta=\sqrt{k}$ for $\rho^{\otimes k}$ in Supplementary Corollary I.2 in Ref. [11], where their $S$ amounts to our system $\mathbf{S}$ and $Q$ is now a trivial system (with dimension 1) because we want to study work extraction on $\mathbf{S}$. Then this corollary implies the existence of a work extraction process $\mathcal{P}_{\rho^{\otimes k}}^{\mathrm{W}}$ such that $\frac{1}{k} W\left(\mathcal{P}_{\rho^{\otimes k}}^{\mathrm{W}}\right) \geq$ $k_{B} T \ln d^{2}-\left[\frac{1}{k} H_{\max }^{\epsilon}\left(\rho^{\otimes k}\right)+\frac{1}{\sqrt{k}}\right] k_{B} T \ln 2$, except with a probability of at most $\sqrt{2^{\frac{-\sqrt{k}}{2}}+12 \epsilon}$. Here we use the notation $H_{\max }^{\epsilon}(\eta)=H_{\max }^{\epsilon}(S \mid Q)_{\eta}$ for the $\epsilon$-smooth maxentropy of $S$ conditional on $Q$ [11]. Using the fact $\lim _{k \rightarrow \infty} \frac{1}{k} H_{\max }^{\epsilon}\left(\rho^{\otimes k}\right)=S(\rho) \forall \epsilon \in(0,1)$, we conclude $\lim _{k \rightarrow \infty} P\left\{\frac{1}{k} W\left(\mathcal{P}_{\rho^{\otimes k}}^{\mathrm{W}}\right) \geq k_{B} T \ln d^{2}-S(\rho) k_{B} T \ln 2-\delta\right\}=$ $1-\sqrt{12 \epsilon}$ for all small enough value $\delta>0$. In other words, this means for every $\delta>0$, there exists $k_{\delta}$ such that $P\left\{\frac{1}{k} W\left(\mathcal{P}_{\rho^{\otimes k}}^{\mathrm{W}}\right) \geq k_{B} T \ln d^{2}-S(\rho) k_{B} T \ln 2-\delta\right\}>$ $1-\sqrt{12 \epsilon}-\delta \forall k \geq k_{\delta}$. For a fixed $\delta$, we let $\epsilon \rightarrow 0$ and obtain $P\left\{\frac{1}{k} W\left(\mathcal{P}_{\rho \otimes k}^{\mathrm{W}}\right) \geq k_{B} T \ln d^{2}-S(\rho) k_{B} T \ln 2-\delta\right\}>1-\delta$ $\forall k \geq k_{\delta}$. This implies $k_{B} T \ln d^{2}-S(\rho) k_{B} T \ln 2-\delta \leq$ $W_{\text {Total }}(\rho)$ for all $\delta>0$. Letting $\delta \rightarrow 0$ will result in the desired inequality.

\section{Appendix D: Proof of Theorem IV.1}

We first prove the following proposition, which is a general version of Theorem IV.1 [recall we define $S_{\min }(\rho):=$ $\left.\min \left\{S\left(\rho_{\mathrm{A}}\right), S\left(\rho_{\mathrm{B}}\right)\right\}\right]$ :

Proposition D.1. Given $0<\epsilon \leq \frac{1}{2}$ and $\delta>\delta_{\epsilon}:=$ $-3 \ln \epsilon$. If

(1) $\mathcal{F}(\rho)>\frac{1}{d}$,

(2) there exists $\mathcal{P}_{\rho}^{\frac{1}{2}-\epsilon}$ which can extract $W_{\text {Total }}(\rho)$, and (3) $-\delta_{\epsilon}<\log _{2}\|\rho\|_{\infty}-\left[\log _{2} \mathcal{F}(\rho) d-S_{\min }(\rho)\right]<\delta-\delta_{\epsilon}$, then we have

$$
\left|\frac{W_{\text {Total }}(\rho)}{k_{B} T \ln 2}-\left[\log _{2} \mathcal{F}(\rho) d^{3}-S_{\min }(\rho)\right]\right|<\delta .
$$

Proof. From Lemma [II.1. Eq. (13), and Refs. [12, 16], we obtain

$$
\begin{aligned}
& k_{B} T \ln d^{2}-\left[H_{\min }^{\epsilon}(\rho)+3 \ln \epsilon\right] k_{B} T \ln 2 \\
& >W_{\text {Total }}(\rho) \geq k_{B} T \ln d^{2}-S(\rho) k_{B} T \ln 2 \\
& \geq k_{B} T \ln \mathcal{F}(\rho) d^{3}-S_{\min }(\rho) k_{B} T \ln 2,
\end{aligned}
$$

where [12]

$$
H_{\min }^{\epsilon}(\rho):=\sup _{\|\eta-\rho\|_{B}<\epsilon}\left(-\log _{2}\|\eta\|_{\infty}\right) \geq-\log _{2}\|\rho\|_{\infty} .
$$


This implies

$$
\begin{aligned}
& \log _{2} d^{2}+\log _{2}\|\rho\|_{\infty}-3 \ln \epsilon>\frac{W_{\text {Total }}(\rho)}{k_{B} T \ln 2} \\
& \geq \log _{2} \mathcal{F}(\rho) d^{3}-S_{\min }(\rho) .
\end{aligned}
$$

Hence, a sufficient condition for the inequality $\left|\frac{W_{\text {Total }}(\rho)}{k_{B} T \ln 2}-\left[\log _{2} \mathcal{F}(\rho) d^{3}-S_{\min }(\rho)\right]\right|<\delta$ reads:

$$
0<\log _{2}\|\rho\|_{\infty}-\left[\log _{2} \mathcal{F}(\rho) d-S_{\min }(\rho)\right]+\delta_{\epsilon}<\delta,
$$

where $\delta_{\epsilon}:=-3 \ln \epsilon>0$ is the best precision that we may have, since it is the lower bound of all the allowed $\delta$-values. Note that being larger than 0 is necessary to guarantee no contradiction.

We are now in position to prove Theorem IV.1

Proof. To begin with, note that $W_{\operatorname{Er}}(\rho) \geq k_{B} T \ln \mathcal{F}(\rho) d$ for state $\rho$ with $\mathcal{F}(\rho)>\frac{1}{d}$ from Theorem 【II.2, and
$W_{\text {Total }}(\rho) \leq \delta k_{B} T \ln 2+k_{B} T \ln 2\left[\log _{2} \mathcal{F}(\rho) d^{3}-S_{\min }(\rho)\right]$ if it is possible to choose $\mathcal{P}_{\rho}^{\frac{1}{2}-\epsilon}$ to extract $W_{\text {Total }}(\rho)$ and $\log _{2}\|\rho\|_{\infty}=\log _{2} \mathcal{F}(\rho) d-S_{\min }(\rho)$ (hence we can choose $\delta \approx \delta_{\epsilon}:=-3 \ln \epsilon$ ), which is due to Theorem IV.1. Then direct computation shows [choose $S_{\min }(\rho)=S\left(\rho_{\mathrm{B}}\right)$ without loss of generality]

$$
\begin{aligned}
0 & \leq W_{\text {Total }}(\rho)-\left[k_{B} T \ln d^{2}-S_{\min }(\rho) k_{B} T \ln 2+W_{\mathrm{Er}}(\rho)\right] \\
& \leq \delta k_{B} T \ln 2+k_{B} T \ln \mathcal{F}(\rho) d-W_{\mathrm{Er}}(\rho) \\
& \leq \delta k_{B} T \ln 2 \approx \delta_{\epsilon} k_{B} T \ln 2
\end{aligned}
$$

where the first inequality follows from the fact that $k_{B} T \ln d^{2}-S_{\min }(\rho) k_{B} T \ln 2+W_{\operatorname{Er}}(\rho)$ can be interpreted as the deterministic work gain (in iid limit) of a particular work extraction process (see Fig. 1), thereby being smaller than the optimal one, i.e. $W_{\text {Total }}(\rho)$. This implies, up to the energy scale $\delta_{\epsilon} k_{B} T \ln 2$, the desired results.
[1] A. Einstein, B. Podolsky, and N. Rosen, Phys. Rev. 47, 777 (1935).

[2] J. S. Bell, Physics 1, 195 (1964).

[3] S. Vinjanampathy and J. Anders, Contemp. Phys. 57, 1 (2016).

[4] R. Landauer, IBM J. Res. Develop. 5, 183 (1961).

[5] R. Alicki, M. Horodecki, P. Horodecki, R. Horodecki, Open Syst. Inf. Dyn. 11, 205 (2004).

[6] M. Horodecki and J. Oppenheim, Nat. Communi. 4, 2059 (2013).

[7] P. Skrzypczyk, A. J. Short, and S. Popescu, Nat. Communi. 5, 4185 (2014).

[8] J. Åberg, Nat. Communi. 4, 1925 (2013).

[9] M. A. Nielson and I. L. Chuang, Quantum Computation and Quantum Information, 10th anniversary ed., Cambridge university press (2010).

[10] P. Faist, F. Dupuis, J. Oppenheim, and R. Renner, Nat. Communi. 6, 7669 (2015).

[11] L. del Rio, J. Åberg, R. Renner, O. Dahlsten, and V. Vedral, Nature 474, 61 (2011).

[12] O. C. O. Dahlsten, R. Renner, E. Rieper, and V. Vedral, New J. Phys. 13, 053015 (2011).

[13] K. V. Hovhannisyan, M. Perarnau-Llobet, M. Huber, and A. Acín, Phys. Rev. Lett. 111, 240401 (2013).

[14] M. Perarnau-Llobet, K. V. Hovhannisyan, M. Huber, P. Skrzypczyk, N. Brunner, and A. Acín, Phys. Rev. X 5, 041011 (2015).

[15] K. Funo, Y. Watanabe, and M. Ueda, Phys. Rev. A 88, 052319 (2013).

[16] J. Oppenheim, M. Horodecki, P. Horodecki, and R. Horodecki, Phys. Rev. Lett. 89, 180402 (2002).

[17] F. N. C. Paraan and A. Silva, Phys. Rev. E 80, 061130 (2009).

[18] L. Fusco, M. Paternostro, and G. De Chiara, Phys. Rev. E 94, 052122 (2016).

[19] V. Hovhannisyan, M. Perarnau-Llobet, M. Huber, and A. Acín, Phys. Rev. Lett. 111, 240401 (2013).

[20] K. Korzekwa, M. Lostaglio, J. Oppenheim, and D. Jen- nings, New J. Phys. 18, 023045 (2016).

[21] A. Misra, U. Singh, S. Bhattacharya, and A. K. Pati, Phys. Rev. A 93, 052335 (2016).

[22] G. L. Giorgy and S. Campbell, J. Phys. B: At. Mol. Opt. Phys. 48, 035501 (2015).

[23] N. Brunner, D. Cavalcanti, S. Pironio, and S. Wehner, Rev. Mod. Phys. 86, 419 (2014).

[24] E. Schrödinger, Proc. Cambridge Philos. Soc. 31, 555 (1935); 32, 446 (1936).

[25] H. M. Wiseman, S. J. Jones, and A. C. Doherty, Phys. Rev. Lett. 98, 140402 (2007).

[26] C. H. Bennett, G. Brassard, C. Crépeau, R. Jozsa, A. Peres, and W. K. Wootters Phys. Rev. Lett. 70, 1895 (1993).

[27] S. Popescu, Phys. Rev. Lett. 72, 797 (1994).

[28] M. Horodecki, P. Horodecki, and R. Horodecki, Phys. Rev. A 60, 1888 (1999).

[29] S. Albeverio, S.-M. Fei, and W.-L. Yang, Phys. Rev. A 66, 012301 (2002).

[30] M.-J. Zhao, Z.-G. Li, S.-M. Fei, and Z.-X. Wang, J. Phys. A: Math. Theor. 43, 27 (2010).

[31] D. Cavalcanti, A. Acín, N. Brunner, and T. Vértesi, Phys. Rev. A 87, 042104 (2013).

[32] C.-Y. Hsieh, Y.-C. Liang, and R.-K. Lee, Phys. Rev. A 94, 062120 (2016).

[33] M. T. Quintino, N. Brunner, and M. Huber, Phys. Rev. A 94, 062123 (2016).

[34] R. Renner, Security of Quantum Key Distribution, Ph.D. dissertation, ETH Zürich, Switzerland (2005).

[35] R. Renner and S. Wolf, Proc. IEEE Int. Symp. Information Theory, 233 (2004).

[36] M. L. Almeida, S. Pironio, J. Barrett, G. Tóth, and A. Acín, Phys. Rev. Lett. 99, 040403 (2007).

[37] R. König, R. Renner, and C. Schaffner, IEEE Trans. Inf. Theory 55, 4337 (2009).

[38] A. Jamiołkowski, Rev. Mod. Phys. 3, 275 (1972).

[39] R. Bhatia, J. Operator Theory 19, 129 (1988).

[40] R. Bhatia and C. Davis, Linear Algebra Appl. 223/224, 
119 (1995). 